Real Analysis Exchange

Vol. 23(2), 1997-1998, pp. 641-651

F. S. Cater, Department of Mathematics, Portland State University,

Portland, Oregon 97207, USA

\title{
ON DERIVATIVES VANISHING ALMOST EVERYWHERE ON CERTAIN SETS
}

\begin{abstract}
Let $g$ be a measurable real valued function on a bounded, measurable subset of the real line. We prove that if $g(E)$ has measure 0 , then 0 is one of the derived numbers of $g$ at almost every point in $E$. We find a function $H$ on the real line that is nondecreasing and closely associated with $G$, such that if $g(E)$ has measure 0 , the $H^{\prime}$ vanishes almost everywhere. Moreover, if $g$ is an $N$-function on $E$ and if $H^{\prime}$ vanishes almost everywhere, then $g(E)$ has measure 0 .
\end{abstract}

1

In this paper $g$ is a measurable function on a bounded measurable set $E$ of real numbers. We let $m$ denote Lebesgue measure and $m_{e}$ denote Lebesgue exterior measure. From $[\mathrm{K}]$ or $[\mathrm{SV}]$ we deduce that if $g$ is differentiable almost everywhere on $E$ and if $m(g(E))=0$, then $g^{\prime}=0$ almost everywhere on $E$. Moreover, if $g$ is an $N$-function (this means $g$ maps subsets of $E$ of measure zero to sets of measure zero) and if $g$ has zero derivative almost everywhere on $E$, then $m(g(E))=0$. These results have application, for example, to variations on the chain rule of differentiation and the change of variables formula of integration (consult $[\mathrm{F}]$ and $[\mathrm{SV}]$ ).

Approximate differentiation [S, chapters VII and IX] is important in real analysis. In section 2 , we prove that these results hold when derivatives are replaced by approximate derivatives. We offer (See also [F, Lemma K] and [El, page 489] the following theorem.

Theorem 2.1. Let g be approximately differentiable almost everywhere on E. We have:

(1) if $m(g(E))=0$, then $g_{a p}^{\prime}=0$ almost everywhere on $E$,

Key Words: derivative, approximate derivative, derived number, $N$-function, measure, bounded variation

Mathematical Reviews subject classification: 26A24, 28A20

Received by the editors December 17, 1997 
(2) if $g$ is an $N$-function on $E$, and if $g_{a p}^{\prime}=0$ almost everywhere on $E$, then $m(g(E))=0$.

We say that a point $x_{o} \in E$ is a knot point of $g$ if $D^{+} g\left(x_{o}\right)=D^{-} g\left(x_{o}\right)=\infty$ and $D_{+} g\left(x_{o}\right)=D_{-} g\left(x_{o}\right)=-\infty$ where $D^{+} g$ denotes the upper right Dini derivative of $g$ relative to $E$, etc.

We deduce from [S, Theorem 10.1, chapter IX] that for almost every $x \in$ $E$, either $x$ is a knot point of $g$ or $g$ is approximately differentiable at $x$. Immediately from Theorem 2.1 we obtain:

Corollary 2.2. Let $m(g(E))=0$. Then almost every $x \in E$ is either a knot point of $g$ or $g_{a p}^{\prime}(x)=0$.

Corollary 2.3. Let $g_{a p}^{\prime} \neq 0$ almost everywhere on $E$, and let $g$ be a one-to-one function on $E$. Then $g^{-1}$ is an $N$-function on $g(E)$.

When we use derived numbers [N, chapter VIII, p. 207] relative to $E$, we can delete the differentiation hypothesis altogether. In section 3 , we offer:

Theorem 3.1. Let $m(g(E))=0$. Then 0 is a derived number of $g$ at almost every $x \in E$.

An immediate consequence of this is:

Corollary 3.2. Let $g$ be one-to-one on $E$, and let all the derived numbers of $g$ be nonzero at almost every $x \in E$. Then $g^{-1}$ is an $N$-function on $g(E)$.

Apparently neither Theorem 2.1(1) nor Theorem 3.1 implies the other, although they each imply part of the result cited in [SV].

In section 4 we try to link zero derivatives with $m_{e}(g(E))$ when $g$ is measurable. The obvious problem is that $g$ need not be differentiable, so we use derivatives of a function closely associated with $g$. For each real number $y$, let $H(y)=m(\{t \in E: g(t)<y\})$. Then $H(y)$ is a nondecreasing function of $y$ mapping $\mathbb{R}$ into the interval $[0, m(E)]$. We offer:

Theorem 4.1. We have:

(1) if $m(g(E))=0$, then $H^{\prime}=0$ almost everywhere on $\mathbb{R}$;

(2) if $H^{\prime}=0$ almost everywhere on $\mathbb{R}$, and if $g$ is an $N$-function on $E$, then $m(g(E))=0$.

We also find use for infinite derivatives. Put

$$
T=\left\{t \in E: m\left(g^{-1}(g(t))\right)=0\right\} .
$$

We offer: 
On Derivatives Vanishing Almost Everywhere on Certain Sets643

Theorem 4.2. We have:

(1) if $m(g(E))=0$, then $H^{\prime}(g(t))=\infty$ for almost every $t \in T$;

(2) if $H^{\prime}(g(t))=\infty$ for almost every $t \in T$, and if $g$ is an $N$-function on $E$, then $m(g(E))=0$.

Now for $t \in E$, put

$$
K(t)=\limsup _{r \downarrow 0} r(H(g(t)+r)-H(g(t)))^{-1} .
$$

We offer:

Theorem 4.3. Let $g$ be an $N$-function on $E$. Then $K$ is a measurable extended real valued function that is finite almost everywhere on E. Moreover,

$$
m(g(E))=\int_{E} K(t) d t .
$$

Thus we found a function $K$ closely associated with $g$ for which equation (*) holds.

2

To prove Theorem 2.1, let $g$ be approximately differentiable almost everywhere on $E$. Say $A=\{x \in E: g$ is approximately differentiable at $x\}$ where $m(E \backslash A)=0$. From $[\mathrm{S}$, Theorem 10.8, chapter VII] we deduce that there is a sequence of sets $A_{1}, A_{2}, A_{3}, \ldots$ where $A=\cup_{n} A_{n}$ and $g$ is of bounded variation on each $A_{n}$. Fix $n$ and let $f$ denote the restriction of $g$ to $A_{n}$. It follows that $f_{A_{n}}^{\prime}(x)=g_{a p}^{\prime}(x)$ at any point of density $x$ of $A_{n}$, and thus $f_{A_{n}}^{\prime}=g_{a p}^{\prime}$ almost everywhere on $A_{n}$.

To prove part (1) assume $m(g(E))=0$. Then $m\left(f\left(A_{n}\right)\right)=0$ and by [SV] we have $f_{A_{n}}^{\prime}=0$ almost everywhere on $A_{n}$. It follows that $g_{a p}^{\prime}=0$ almost everywhere on $A_{n}$. But $n$ was arbitrary, so $g_{a p}^{\prime}=0$ almost everywhere on $A$ and on $E$.

To prove part (2) assume $g$ is an $N$-function on $E$ and $g_{a p}^{\prime}=0$ almost everywhere on $E$. Let $f$ and $A_{n}$ be as in the preceding paragraph. Then $f$ is an $N$-function on $A_{n}$ and $f_{A_{n}}^{\prime}=0$ almost everywhere on $A_{n}$. By [SV] we have $m\left(f\left(A_{n}\right)\right)=0$ and hence $m\left(g\left(A_{n}\right)\right)=0$. But $n$ is arbitrary, so $m(g(A))=0$. Finally, $m(E \backslash A)=m(g(E \backslash A))=0$ because $g$ is an $N$-function on $E$. It follows that $m(g(E))=0$. 
The proof of Corollary 2.2 was essentially given in section 1 , so we omit it here.

To prove Corollary 2.3 , let $B$ be a subset of $g(E)$ with $m(B)=0$. There is a set $C$ that is the intersection of countably many open sets in $\mathbb{R}$ such that $B \subset C$ and $m(C)=0$. Then $g^{-1}(C)$ is measurable because $g$ is a measurable function on $E$. By Theorem 2.1, $g_{a p}^{\prime}=0$ almost everywhere on $g^{-1}(C)$ and from the hypothesis we deduce that $m\left(g^{-1}(C)\right)=0$. But $B \subset C$ so $m\left(g^{-1}(B)\right)=0$.

3

We begin this section with a lemma that may be of some interest in its own right.

Lemma I. Let $p \geq 1$ and let $m(E)>p^{2} m_{e}(g(E))$. Then there is a measurable subset $A$ of $E$ such that $m(A)>\left(1-p^{-1}\right) m(E)$ and for each $x \in A$ there is $a u \in E$ (depending on $x$ ) with $|g(x)-g(u)|<2 p^{-1}|x-u|$.

Proof. Let $I_{1}, I_{2}, I_{3}, \ldots$ be a sequence of mutually disjoint open intervals covering $g(E)$ such that $\sum_{n} m\left(I_{n}\right)<p^{-2} m(E)$. Let $J_{1}, J_{2}, J_{3}, \ldots$ be those intervals $I_{n}$ for which $m\left(g^{-1}\left(I_{n}\right)\right)>p \cdot m\left(I_{n}\right)$, and let $K_{1}, K_{2}, K_{3}, \ldots$ be the remaining $I_{n}$. Now $g$ is measurable, so

$$
m\left(\cup_{j} g^{-1}\left(K_{j}\right)\right)=\sum_{j} m\left(g^{-1}\left(K_{j}\right)\right) \leq p \cdot \sum_{j} m\left(K_{j}\right)
$$

by the choice of the $K_{j}$. But $\sum_{j} m\left(K_{j}\right) \leq \sum_{n} m\left(I_{n}\right)<p^{-2} m(E)$, so

$$
m\left(\cup_{j} g^{-1}\left(K_{j}\right)\right)<p^{-1} m(E) .
$$

Also by (1),

so

$$
\begin{gathered}
m(E)=m\left(\cup_{n} g^{-1}\left(I_{n}\right)\right)=m\left(\cup_{j} g^{-1}\left(J_{j}\right)\right)+m\left(\cup_{j} g^{-1}\left(K_{j}\right)\right)< \\
<m\left(\cup_{j} g^{-1}\left(J_{j}\right)\right)+p^{-1} m(E),
\end{gathered}
$$

$$
m\left(\cup_{j} g^{-1}\left(J_{j}\right)\right)>\left(1-p^{-1}\right) m(E) .
$$

It remains to prove that $\cup_{j} g^{-1}\left(J_{j}\right)$ suffices for $A$. Let $x \in \cup_{j} g^{-1}\left(J_{j}\right)$. Say $x \in g^{-1}\left(J_{N}\right)$ and $g(x) \in J_{N}$. Recall that by the choice of $J_{N}$,

$$
m\left(g^{-1}\left(J_{N}\right)\right)>p \cdot m(E) .
$$


On Derivatives Vanishing Almost Everywhere on Certain Sets645

There are points $u, v \in g^{-1}\left(J_{N}\right)$ such that

$$
|u-v|>p \cdot m\left(J_{N}\right) .
$$

Moreover, $g(u), g(v) g(x) \in J_{N}$ and because $J_{N}$ is an open interval,

$$
|g(x)-g(u)|<m\left(J_{N}\right) \text { and }|g(x)-g(v)|<m\left(J_{N}\right) .
$$

Now by (4), $|x-u|+|x-v| \geq|u-v|>p \cdot m\left(J_{N}\right)$, so either $|x-u|>p \cdot m\left(J_{N}\right) / 2$ or $|x-v|>p \cdot m\left(J_{N}\right) / 2$. Then by (5), either $|x-u|>p|g(x)-g(u)| / 2$ or $|x-v|>p|g(x)-g(v)| / 2$.

To prove Theorem 3.1, for each positive integer $i$ partition $E$ into finitely many mutually disjoint measurable sets $E_{i 1}, E_{i 2}, E_{i 3}, \ldots$, each of diameter $<2^{-i}$. By hypothesis, $m\left(g\left(E_{i j}\right)\right)=0$ for all $i$ and $j$. We deduce from Lemma I, there is measurable set $A_{i j} \subset E_{i j}$ such that $m\left(E_{i j} \backslash A_{i j}\right)<2^{-i-j} m\left(E_{i j}\right)$ and for each $x \in A_{i j}$ there is a $u \in E_{i j}$ with $|g(x)-g(u)|<2^{-i}|x-u|$. We leave the proof that 0 is a derived number of $g$ at each point in $B=\cap_{k=1}^{\infty} \cup_{i=k}^{\infty} \cup_{j} A_{i j}$ and $m(E \backslash B)=0$.

The proof of Corollary 3.2 is analogous to the proof of Corollary 2.3, so we leave it.

4

We begin with a lemma to dispose of certain details.

Lemma II. If $S \subset T$, and $m(H(g(S)))=0$, then $m(S)=0$. Moreover, if at each $x \in S$ either $H^{\prime}(g(x))=0$ or $H$ does not have a finite or infinite derivative at $g(x)$, then $m(S)=0$.

Proof. Let $m(H(g(S)))=0$. Choose $\epsilon>0$. Let $\left(a_{1}, b_{1}\right),\left(a_{2}, b_{2}\right),\left(a_{3}, b_{3}\right), \ldots$ be a sequence of open intervals covering $H(g(S))$ with $\sum_{i}\left(b_{i}-a_{i}\right)<\epsilon$. Thus

$$
H(g(S)) \subset \cup_{i}\left(a_{i}, b_{i}\right) .
$$

For each index $i$, put $S_{i}=\left\{s \in S: H(g(s)) \in\left(a_{i}, b_{i}\right)\right\}$. Let $u_{1}, u_{2} \in S_{i}$ for some index $i$, where $H\left(g\left(u_{1}\right)\right) \leq H\left(g\left(u_{2}\right)\right)$. Then $a_{i}<H\left(g\left(u_{1}\right)\right) \leq H\left(g\left(u_{2}\right)\right)<$ $b_{i}$. So

$$
a_{i}<m\left\{t \in E: g(t)<g\left(u_{1}\right)\right\} \leq m\left\{t \in E: g(t)<g\left(u_{2}\right)\right\}<b_{i} .
$$

Because $g$ is measurable,

$$
\begin{aligned}
& m\left\{t \in E: g\left(u_{1}\right) \leq g(t)<g\left(u_{2}\right)\right\} \\
= & m\left\{t \in E: g(t)<g\left(u_{2}\right)\right\}-m\left\{t \in E: g(t)<g\left(u_{1}\right)\right\} .
\end{aligned}
$$


So

$$
\left\{t \in E: g\left(u_{1}\right) \leq g(t)<g\left(u_{2}\right)\right\}<b_{i}-a_{i} .
$$

But $u_{2} \in T$, so $m\left\{t \in E: g(t)=g\left(u_{2}\right)\right\}=0$, and by $(2)$

$$
m\left\{t \in E: g\left(u_{1}\right) \leq g(t) \leq g\left(u_{2}\right)\right\}<b_{i}-a_{i} .
$$

It is not difficult to see that

$$
m\left(S_{i}\right) \leq\left(b_{i}-a_{i}\right) .
$$

(Just let $g\left(u_{1}\right)$ tend to $\inf g\left(S_{i}\right)$ and $g\left(u_{2}\right)$ tend to $\sup g\left(S_{i}\right)$, etc.)

It follows from (1) and (4) that

$$
m(S) \leq \sum_{i} m\left(S_{i}\right) \leq \sum_{i}\left(b_{i}-a_{i}\right)<\epsilon .
$$

Finally, $\epsilon$ is arbitrary, so $m(S)=0$. Put

$$
\begin{aligned}
& S^{\prime}=\left\{s \in S: H^{\prime}(g(s))=0\right\}, \\
& S^{\prime \prime}=\{s \in S: H \text { has no finite or infinite derivative at } g(s)\} .
\end{aligned}
$$

Then $m\left(H\left(g\left(S^{\prime}\right)\right)\right)=0$. By de la Vallée Poussin's Theorem (see for example [S, Theorem (9.1), chapter IV]), we see that $m\left(H\left(g\left(S^{\prime \prime}\right)\right)\right)=0$.

To prove the second statement in Lemma II, assume $S=S^{\prime} \cup S^{\prime \prime}$. Hence $m(H(g(S))) \leq m\left(H\left(g\left(S^{\prime}\right)\right)\right)+m\left(H\left(g\left(S^{\prime \prime}\right)\right)\right)=0$. So $m(H(g(S)))=0$. By the previous part, $m(S)=0$.

We turn now to the theorems in section 4 .

Proof of Theorem 4.1(1). Let $m(g(E))=0$. Let $\epsilon>0$. Let $I_{1}, I_{2}, I_{3}, \ldots$ be mutually disjoint open intervals covering $g(E)$ such that $\sum_{j} m\left(I_{j}\right)<\epsilon$. Select an index $N$ so that $\sum_{j=N+1}^{\infty} m\left(g^{-1}\left(I_{j}\right)\right)<\epsilon^{2}$. Then

$$
m\left(\cup_{j=N+1}^{\infty} g^{-1}\left(I_{j}\right)\right)<\epsilon^{2} .
$$

Let $\left[a_{1}, b_{1}\right],\left[a_{2}, b_{2}\right],\left[a_{3}, b_{3}\right], \ldots$ be mutually disjoint closed intervals, each disjoint from $\cup_{j=1}^{N} I_{j}$. By $(6)$ and the definition of $H$, we have $\sum_{j}\left(H\left(b_{j}\right)-\right.$ $\left.H\left(a_{j}\right)\right)<\epsilon^{2}$. By [HS, Theorem (18.14), chapter V],

$$
\sum_{j} \int_{a_{j}}^{b_{j}} H^{\prime}(x) d x \leq \sum_{j}\left(H\left(b_{j}\right)-H\left(a_{j}\right)\right)<\epsilon^{2} .
$$


On Derivatives Vanishing Almost Everywhere on Certain Sets647

Let $D=\left\{x: H^{\prime}(x)>\epsilon\right\}$. We deduce from (7) that

$$
m\left(D \cap\left(\cup_{j}\left[a_{j}, b_{j}\right]\right)\right)<\epsilon .
$$

From (8) we deduce that $m\left(D \backslash\left(\cup_{j=1}^{N} I_{j}\right)\right) \leq \epsilon$. But $m\left(\cup_{j=1}^{N} I_{j}\right)<\epsilon$, so

$$
m(D)<2 \epsilon .
$$

Because $\epsilon$ is arbitrary, we conclude that $m\left\{x: H^{\prime}(x)>0\right\}=0$.

Proof of Theorem 4.1(2). Let $H^{\prime}=0$ almost everywhere, and let $g$ be an $N$-function on $E$. Let $S=\left\{s \in T: H^{\prime}(g(s))=0\right\}$. By Lemma II, $m(S)=0$. Because $g$ is an $N$-function on $E, m(g(S))=0$. Now $H^{\prime}=0$ almost everywhere, so $m(g(T \backslash S))=0$, and hence $m(g(T))=0$.

Moreover, $g^{-1}(y)$ can have positive measure for at most countably many $y$, so $g(E \backslash T)$ is countable. Finally, $m(g(E))=0$.

Proof of Theorem 4.2(1). Let $m(g(E))=0$. Put

$T_{1}=\{t \in T: H$ has no finite or infinite derivative at $g(t)\}$,

$T_{2}=\{t \in T: H$ has a finite derivative at $g(t)\}$.

By Lemma II, $m\left(T_{1}\right)=0$. We deduce from [S, Theorem (4.5), chapter IX] and $m\left(g\left(T_{2}\right)\right)=0$ that $m\left(H\left(g\left(T_{2}\right)\right)=0\right.$. By Lemma II, $m\left(T_{2}\right)=0$. So $m\left(T_{1} \cup T_{2}\right)=0$, and $t \in T \backslash\left(T_{1} \cup T_{2}\right)$ implies $H^{\prime}(g(t))=\infty$.

Proof of Theorem 4.2(2). Let $H^{\prime}(g(t))=\infty$ for almost every $t \in T$ and let $g$ be an $N$-function on $E$. Put $T_{0}=\left\{t \in T: H^{\prime}(g(t))=\infty\right\}$. Then $m\left(g\left(T_{0}\right)\right)=0$ by $\left[\mathrm{S}\right.$, Theorem (4.4), chapter IX]. But $m\left(T \backslash T_{0}\right)=0$ by hypothesis and hence $m\left(g\left(T \backslash T_{0}\right)\right)=0$ because $g$ is an $N$-function on $E$. It follows that $m(g(T))=0$. We recall that $g(E \backslash T)$ is a countable set, so $m(g(E))=0$.

For our last result, we need more lemmas.

Lemma III. Let $g$ be an $N$-function on $E$. Then there exists a measurable set $P \subset T$ such that

(i) $K(t)=0$ for almost every $t \in E \backslash P$,

(ii) $m(g(E \backslash P))=0$,

(iii) $0<K(t)=1 / H^{\prime}(g(t))<\infty$ for every $t \in P$. 
Proof. If $t \in E \backslash T$, it follows that

$$
H(g(t)+h)-H(g(t)) \geq m\left(g^{-1}(g(t))\right)>0
$$

for any $h>0$, and it follows from the definition of $K$ that $K(t)=0$. We recall that $g(E \backslash T)$ is countable, so $m(g(E \backslash T))=0$. Now put

$$
\begin{aligned}
& T_{3}=\{t \in T: H \text { has no finite or infinite derivative at } g(t)\}, \\
& T_{4}=\left\{t \in T: H^{\prime}(g(t))=0\right\} \\
& T_{5}=\left\{t \in T: H^{\prime}(g(t))=\infty\right\}
\end{aligned}
$$

By Lemma II, $m\left(T_{3}\right)=m\left(T_{4}\right)=0$, and because $g$ is an $N$-function on $E$, $m\left(g\left(T_{3}\right)\right)=m\left(g\left(T_{4}\right)\right)=0$. For $t \in T \backslash\left(T_{3} \cup T_{4}\right)$ it follows that $H$ has a positive finite or infinite derivative at $g(t)$, and it follows from the definition of $K$ that $K(t)=1 / H^{\prime}(g(t))$ (here $\left.0=1 / \infty\right)$. But $H(g(t))$ and $H(g(t)+h)$ are measurable functions of $t$ because $g$ is measurable and $H$ is monotonic. We deduce that $K$ is measurable on $T \backslash\left(T_{3} \cup T_{4}\right)$. Then $T_{5}$ is a measurable set. By [S, Theorem (4.4), chapter IX], $m\left(g\left(T_{5}\right)\right)=0$. By the definition of $K$, $K(t)=0$ for any $t \in T_{5}$.

Put $P=T \backslash\left(T_{3} \cup T_{4} \cup T_{5}\right)$. Then $P$ is measurable because $T$ and the $T_{i}$ are measurable. Finally, (i), (ii) and (iii) follow from the preceding paragraph.

It is well-known that if $g$ is a measurable $N$-function on $E$, then $g(E)$ is measurable. It follows that $g(P)$ is measurable in Lemma III.

Lemma IV. Let $g$ be an $N$-function on $E$. Let $c, d, u$ be real numbers such that $u>0$ and $0<c<d$. Let $L$ be a closed set such that for every $x \in L$ and $y$ satisfying $x<y<x+u$, we have $c(y-x) \leq H(y)-H(x) \leq d(y-x)$. Then

$$
c \cdot m(L) \leq m\left(g^{-1}(L)\right) \leq d \cdot m(L) .
$$

Proof. Let $n$ be an integer with $n^{-1}<u$. Cover $L$ with countably many mutually disjoint half open intervals $\left[a_{1}, b\right),\left[a_{2}, b_{2}\right),\left[a_{3}, b_{3}\right), \ldots$ so that $b_{i}-a_{i}<$ $n^{-1}$ and $a_{i} \in L$ for each $i$. Let $U_{n}=\cup_{i}\left[a_{i}, b_{i}\right)$. It follows that

$$
c\left(b_{i}-a_{i}\right) \leq H\left(b_{i}\right)-H\left(a_{i}\right) \leq d\left(b_{i}-a_{i}\right) \text { for each } i,
$$

and hence

$$
c\left(b_{i}-a_{i}\right) \leq m\left(g^{-1}\left[a_{i}, b_{i}\right)\right) \leq d\left(b_{i}-a_{i}\right) .
$$

It follows that

$$
c \cdot \sum_{i}\left(b_{i}-a_{i}\right) \leq \sum_{i} m\left(g^{-1}\left(\left[a_{i}, b_{i}\right)\right)\right) \leq d \cdot \sum_{i}\left(b_{i}-a_{i}\right)
$$


On Derivatives Vanishing Almost Everywhere on Certain Sets649

and

$$
c \cdot m\left(U_{n}\right) \leq m\left(g^{-1}\left(U_{n}\right)\right) \leq d \cdot m\left(U_{n}\right) .
$$

By inductive construction, we choose $U_{n}$ so that $U_{n} \subset U_{n-1}$ for all $n>1+u^{-1}$. The distance from the closed set $L$ to any point in $U_{n}$ cannot exceed $n^{-1}$. Hence $\cap_{n} U_{n}=L$. From (11) we deduce $c \cdot m(L) \leq m\left(g^{-1}(L)\right) \leq d \cdot m(L)$.

Lemma V. Let $g$ be an $N$-function on $E$. Let $c, d, u$ be real numbers such that $u>0$ and $0<c<d$. Let $L_{1}=\{x \in g(P)$ : for any $y$ such that $x<y<x+u$ we have $c(y-x) \leq H(y)-H(x) \leq d(y-x)\}$. Then

$$
c \cdot m\left(L_{1}\right) \leq m\left(g^{-1}\left(L_{1}\right)\right) \leq d \cdot m\left(L_{1}\right) .
$$

Proof. If $x \in g(P)$ and $x$ is in the closure of $L_{1}$ and if $H$ is continuous at $x$, it is easy to see that $x \in L_{1}$. So we leave the proof that $L_{1}$ is measurable. Say $L_{1}=M_{0} \cup M_{1} \cup M_{2} \cup M_{3} \cup \ldots$ where $m\left(M_{0}\right)=0, M_{1} \subset M_{2} \subset M_{3} \subset \ldots$, and each $M_{i}(i>0)$ is closed. Now $M_{0} \subset g(P)$ and because $H$ is differentiable at each point of $M_{0}$, we have $m\left(H\left(M_{0}\right)\right)=0$. Then by Lemma II, $m\left(g^{-1}\left(M_{0}\right)\right)=$ 0 . By Lemma IV, $c \cdot m\left(M_{i}\right) \leq m\left(g^{-1}\left(M_{i}\right)\right) \leq d \cdot m\left(M_{i}\right)$ for each $i>0$. It follows that

$$
\begin{gathered}
c \cdot m\left(M_{0} \cup M_{1} \cup M_{2} \cup \ldots\right) \leq m\left(g^{-1}\left(M_{0} \cup M_{1} \cup M_{2} \cup \ldots\right)\right) \leq \\
\leq d \cdot m\left(M_{0} \cup M_{1} \cup M_{2} \cup \ldots\right),
\end{gathered}
$$

or in other words $c \cdot m\left(L_{1}\right) \leq m\left(g^{-1}\left(L_{1}\right)\right) \leq d \cdot m\left(L_{1}\right)$.

In the next lemma, we can see the proof of Theorem 4.3 emerging.

Lemma VI. Let $g$ be an $N$-function on $E$. Let $c, d$ be real numbers such that $0<c<d$ and let $V=\left\{x \in g(P): c<H^{\prime}(x)<d\right\}$. Then

$$
c \cdot m(V) \leq m\left(g^{-1}(V)\right) \leq d \cdot m(V) .
$$

Proof. For indices $i, j$, put $V_{i j}=\{x \in V$ : for any $y$ such that $x<y<$ $x+i^{-1}$, we have $\left.\left(c+j^{-1}\right)(y-x) \leq H(y)-H(x) \leq\left(d-j^{-1}\right)(y-x)\right\}$. By Lemma $\mathrm{V}$, we have for each $i$ and $j$,

$$
\left(c+j^{-1}\right) \cdot m\left(V_{i j}\right) \leq m\left(g^{-1}\left(V_{i j}\right)\right) \leq\left(d-j^{-1}\right) \cdot m\left(V_{i j}\right) .
$$

For each $j, V_{1 j} \subset V_{2 j} \subset V_{3 j} \subset \ldots$ and we deduce from (12) that for each $j$,

$$
\left(c+j^{-1}\right) \cdot m\left(\cup_{i} V_{i j}\right) \leq m\left(g^{-1}\left(\cup_{i} V_{i j}\right)\right) \leq\left(d-j^{-1}\right) \cdot m\left(\cup_{i} V_{i j}\right) .
$$

Moreover $\cup_{i} V_{i 1} \subset \cup_{i} V_{i 2} \subset \cup_{i} V_{i 3} \subset \cup \ldots$ and we deduce from (13) that

$$
c \cdot m\left(\cup_{i} \cup_{j} V_{i j}\right) \leq m\left(g^{-1}\left(\cup_{i} \cup_{j} V_{i j}\right)\right) \leq d \cdot m\left(\cup_{i} \cup_{j} V_{i j}\right) .
$$

Finally, $\cup_{i} \cup_{j} V_{i j}=V$. 
Proof of Theorem 4.3. Let $g$ be an $N$-function on $E$. Choose $\epsilon>0$. Let $y_{0}, y_{1}, y_{-1}, y_{2}, y_{-2}, y_{2}, y_{-3}, \ldots$ be positive numbers such that $0<y_{i}-y_{i-1}<\epsilon$, $m\left(\left\{t \in P: K(t)=y_{i}\right\}\right)=0$ for each index $i$, and

$$
\lim _{i \rightarrow-\infty} y_{i}=0, \quad \lim _{i \rightarrow \infty} y_{i}=\infty .
$$

Let $P_{i}=\left\{t \in P: y_{i-1}<K(t)<y_{i}\right\}$ for each $i$. By Lemma III, $y_{i}^{-1}<$ $H^{\prime}(g(t))<y_{i-1}^{-1}$ for $t \in P_{i}$. By Lemma VI and the definition of $P_{i}$, we have $P_{i}=g^{-1}\left(g\left(P_{i}\right)\right)$ and

$$
y_{i}^{-1} \cdot m\left(g\left(P_{i}\right)\right) \leq m\left(P_{i}\right) \leq y_{i-1}^{-1} m\left(g\left(P_{i}\right)\right) .
$$

This can be rewritten

$$
y_{i-1} \cdot m\left(P_{i}\right) \leq m\left(g\left(P_{i}\right)\right) \leq y_{i} \cdot m\left(P_{i}\right) .
$$

But also

$$
y_{i-1} \cdot m\left(P_{i}\right) \leq \int_{P_{i}} K(t) d t \leq y_{i} \cdot m\left(P_{i}\right)
$$

and we combine these inequalities to obtain

$$
\left|m\left(g\left(P_{i}\right)\right)-\int_{P_{i}} K(t) d t\right| \leq\left(y_{i}-y_{i-1}\right) \cdot m\left(P_{i}\right)<\epsilon \cdot m\left(P_{i}\right) .
$$

We sum to obtain

$$
\left|\sum_{i=-\infty}^{\infty}\left(m\left(g\left(P_{i}\right)\right)-\int_{P_{i}} K(t) d t\right)\right| \leq \epsilon \cdot \sum_{i=-\infty}^{\infty} m\left(P_{i}\right)=\epsilon \cdot m(P) .
$$

It follows from (15) that $\left|m(g(P))-\int_{P} K(t) d t\right| \leq \epsilon \cdot m(P)$ if $m(g(P))<\infty$, and $\int_{P} K(t) d t=\infty$ if $m(g(P))=\infty$. Because $\epsilon$ is arbitrary we conclude that in any case

$$
m(g(P))=\int_{P} K(t) f t .
$$

In view of Lemma III, equation $(*)$ follows from $(16)$.

\section{References}

[El] H. W. Ellis, Darboux properties and applications to non-absolute convergent integrals , Canad. J. Math., 3 (1951), 471-485. 
On Derivatives Vanishing Almost Everywhere on Certain Sets651

[En] V. Ene, Real functions-current topics , Lect. Notes in Math., vol. 1603, Springer-Verlag, 1995.

[F] J. Foran, A chain rule for the approximate derivative and change of variables for the (D)-integral, Real Analysis Exch., 8 (1982-83), 443454.

[HS] E. Hewitt and K. Stromberg, Real and abstract analysis, Springer Verlag,

[K] K. Krzyzewski, On change of variable in the Denjoy-Perron integral (I), (II) , Coll. Math., 9 (1962), 99-104 and 317-323. 1965.

[N] I. P. Natanson, Theory of functions of a real variable, Ungar, New York, 1955.

[S] S. Saks, Theory of the integral, 2nd. rev. ed., Dover, New York, 1964.

[SV] J. Serrin and D. E. Varberg, A general chain rule for derivatives and the change of variable formula for the Lebesgue integral, Amer. Math. Monthly 76 (1969), 514-520. 